\title{
In vitro \& in vivo investigation of the silicon nitride ceramic hip implant's safety and effectiveness evaluation
}

\author{
Xiangpeng Kong ${ }^{1}$, Xiaosu $\mathrm{Hu}^{2}$ and Wei Chai ${ }^{*}$ (1)
}

\begin{abstract}
Background: With regard to the ceramic hip joint implant, given the concerns in ceramic about the alumina brittleness and zirconia instability, is there any alternative material solution for the orthopedic implant? Beyond the metastable oxide ceramics, along the echelon of advanced technical ceramics, looking at the non-oxide ceramic, the silicon nitride could be an excellent candidate for the joint implant's application. The purpose of this study is to investigate the safety, effectiveness and to demonstrate the potential of this silicon nitride hip implant.
\end{abstract}

Methods: According to the related ISO (International Organization for Standardization) standards, a series of in vitro (nine) \& in vivo (five) tests, which had been accomplished for the aforementioned aim. Especially, the total hip replacement in pigs had been achieved, as per the authors' knowledge, this is the first time to apply the THA (Total Hip Arthroplasty) in the big animal.

Results: Refer to the ISO 6474-2, in comparison with the current monopolized German product, this silicon nitride ceramic hip implant has high strength, high hardness, excellent fracture toughness, lower density, better wear resistance, good biocompatibility, inherent stability, corrosion resistance and bioactivity, bone integration capability.

Conclusions: This silicon nitride ceramic will be an admirable alternative solution with superior comprehensive property that can withstand the toughest conditions in the most demanding applications like in orthopedic and beyond.

Keywords: Silicon nitride, Ceramic femoral head, Hip replacement, In vitro \& in vivo experiments

\section{Introduction}

With the increasing trend of Chinese aging population and the continuous improvement of life expectancy and living standards, people's awareness of healthy life has changed, currently Total Hip Arthroplasty (THA) is gradually admitted and accepted in China, it is also usually recognized as one of the most successful modern surgery [1].

Meanwhile, with regard to the avascular necrosis of the femoral head, the demands from the relative young

\footnotetext{
*Correspondence: chaiweiguanjie@sina.com

1 Senior Department of Orthopedics, The Fourth Medical Center of PLA general hospital, No. 51 Fucheng Road, Haidian, Beijing 100853, China Full list of author information is available at the end of the article
}

patients have been raised due to the alcoholic or sportive traumata or genetic predilection. As the last resort, THA will restore these patients' health [2].

A safe, effective and durable hip implant is desirable consequently.

In terms of the material improvement, compared with John Charnley's metal femoral head [3], the introduction of ceramic in THA was a great initiation since almost five decades ago [4].

Considering the appreciated excellent biological behavior, no metal ion release, no known pathogenic reaction to particles, excellent wettability, resistant to third-body wear, significantly low taper corrosion etc., thanks to these inherent proprieties of ceramic, more than 18 
million oxide ceramic components are sold since 1974 by the monopolized German giant [5].

However, the poor toughness is the biggest demerit of the alumina ceramic [6], in order to solve the problem of alumina brittleness, zirconia, one of the highest strength ceramics, has been wildly used as femoral head since $1985[7,8]$.

Nevertheless, this metastable zirconia ceramic for medical use were suspended on August 14, 2001 by SaintGobain Desmarquest (Vincennes, France) and a Medical Device Recall Notice was issued one month later by the FDA (U. S. Food and Drug Administration) [9] due to the catastrophic experience with Prozyr ${ }^{\circledR}$ femoral head produced by this world's largest supplier of medical-grade stabilized zirconia ceramic [10].

The root cause of this failure is the inherent zirconia phase transformation. By a stress corrosion mechanism, the initial phase transformation of isolated grains occurs on the machined and polished surfaces, where is not only the femoral head bearing surface, but also the inner taper surface in contact with the femoral stem. The zirconia transforms from metastable tetragonal to monoclinic phase, this phase change results a volumetric expansion in the grains that induces a compressive stress increase and leads to the propagation of the micro cracks and surface roughened [11].

Under more severe environment, especially with presence of moisture, like in the human body, this zirconia intrinsic aging process will transform more aggressively to the monoclinic phase with catastrophic consequence: increased wear, grain pull-out and generation of particle debris even through the premature failure [12].

Given the concerns about the monolithic alumina brittleness and zirconia instability, to counteract these deficiencies, different approaches have been proposed like developing alumina-zirconia composites: ZTA (Zirconia Toughened Alumina) or replacing yttria with other additives such as ceria to stabilize zirconia.

Although zirconia transformation toughening effect is well appreciated, it is also exceedingly complex and still not totally understood.

Clarke et al. [11] and Chevalier [12] tried to pierce the veil of mystique surrounding zirconia. The potential effect of different process stages on microstructure of zirconia and consequently on phase transformation and aging were summarized. And it is remarkable that that ageing could be significant in the alumina-3Y-TZP (Yttria-stabilized Tetragonal Zirconia Polycrystal) composite above 16 vol\% zirconia. This critical content was related to the percolation threshold above which a continuous path of zirconia grains allowed transformation to proceed. The latter author also emphasized that, for alumina-zirconia composite, the presence of zirconia aggregates, especially if the zirconia is stabilized with yttria, should be avoided [12].

From some orthopedic surgeons' point of view, it is unsatisfactory to implant a material into the body, which is not fully stable.

In this respect, beyond the metastable oxide ceramics, along the echelon of advanced technical ceramics, looking at the non-oxide ceramic, the silicon nitride could be a suitable alternative material solution for joint implants.

The purpose of this paper is to investigate the silicon nitride hip implant's safety and effectiveness via in vitro and in vivo experiments then to demonstrate the applicability and potential of this silicon nitride ceramic in the orthopedic domain.

\section{Materials and methods}

In this study, a series of in vitro (nine) \& in vivo (five) tests had been designed, 5 in vitro tests were descriptive, the rest other 4 in vitro tests and 5 in vivo tests were analytic. And, the level of evidence is Level I since the 5 in vivo tests were all randomized controlled trials.

The approval was obtained from the Institutional Review Board of Chinese PLA General Hospital prior to performing the study.

\section{Specimens tested}

According to the specific requirements defined by the related ISO standards, the relevant silicon nitride specimens were prepared for two categories experimentation: in vitro \& in vivo experiments. Both sample bars (Fig. 1) and femoral heads (Fig. 2) were produced via the patented material formula and technological process by Black Ceramic Medical Technology Co., Ltd. (Suzhou, China). The geometrical dimension and tolerance of the femoral head are detailed in Fig. 3.

As per the animal CT (Computed Tomography) scan data, the femoral stem was customized in wrought cobalt-chromium-molybdenum alloy by $3 \mathrm{D}$ printing from LDK Technology Co., Ltd. (Beijing, China), the cemented acetabular cup was made of UHMWPE (Fig. 4).

Thirteen health Bama minipigs (Fig. 5) were purchased from Beijing Strong Century Minipigs Breeding Base. Minipigs were fed in Laboratory Animal Center of Chinese PLA (People's Liberation Army of China) General Hospital (Beijing, China), with a 12:12-h light-dark cycle, room temperature maintained between 20 and $22{ }^{\circ} \mathrm{C}$ and humidity range $50-60 \%$, for one week prior to experiment in order to adapt to environment. The Bama minipigs' weight was between 25 and $29 \mathrm{~kg}$. All animal care and procedures were approved by the Animal Care Committee of Chinese PLA General Hospital and complied with the latest guideline. 

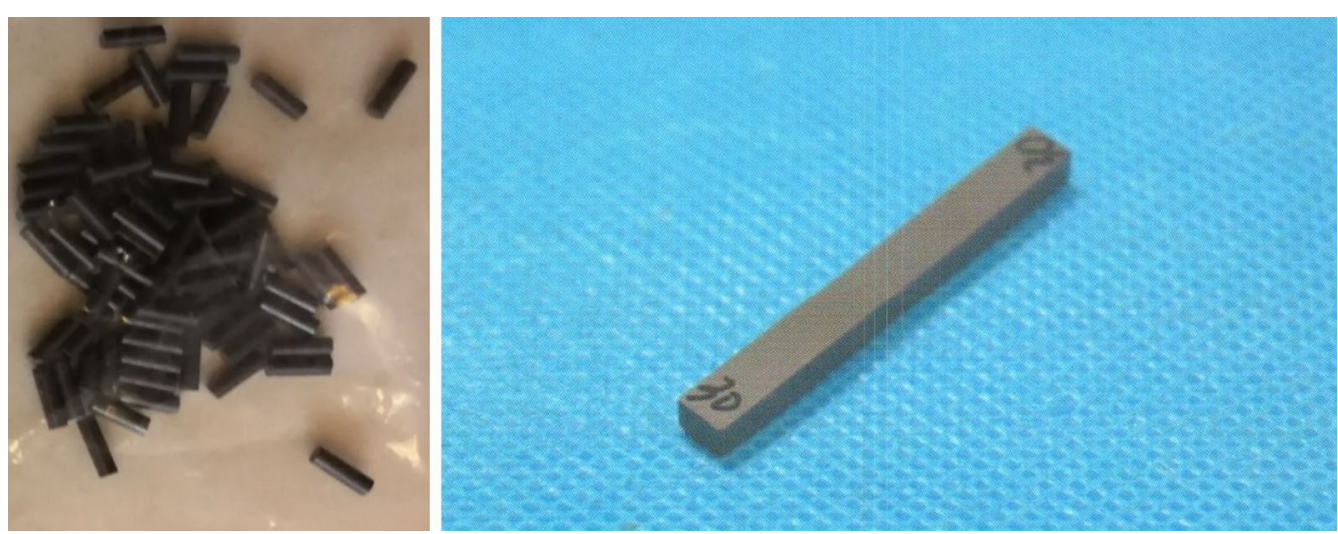

Fig. 1 Sample bars $(\Phi 2 * 6 \mathrm{~mm} \& 3 * 4 * 50 \mathrm{~mm})$
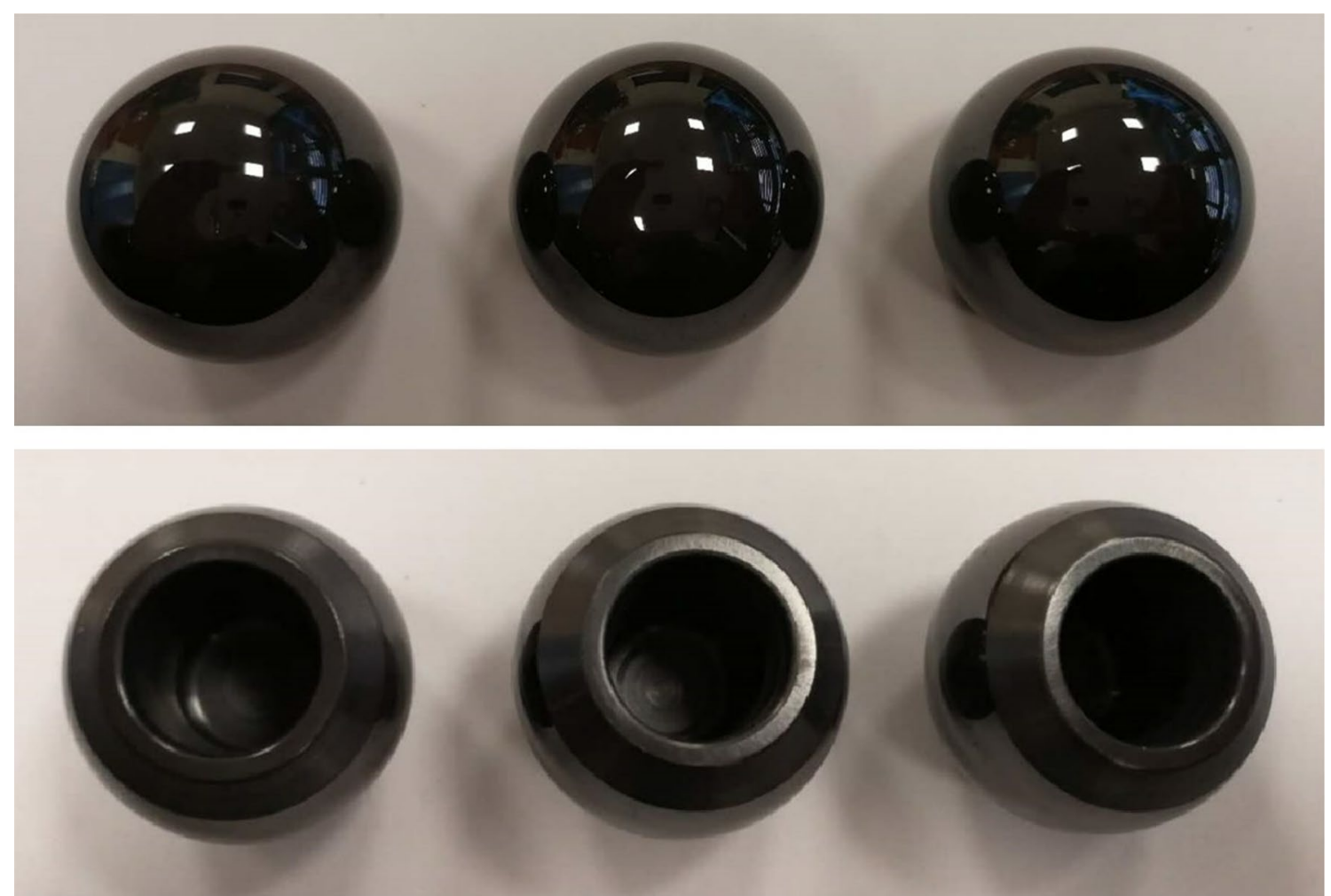

Fig. 2 Femoral heads (Ф28 mm)

\section{In vitro experiments}

Accelerated ageing test

Within the domain of hip implants, the accelerated ageing is usually a test method for investigating the oxidative stability of Ultra-High-Molecular-WeightPolyethylene (UHMWPE) materials as a function of processing and sterilization method. The UHMWPE is aged at elevated temperature and at elevated oxygen pressure, to accelerate oxidation of the material and thereby allow for the evaluation of its potential longterm chemical and mechanical stability. The related principle, process and requirements are calibrated in (ISO 5834-3).

However, with regard to the ceramics applied as orthopedic prostheses, especially for the metastable oxide ceramics like zirconia or its composites, 

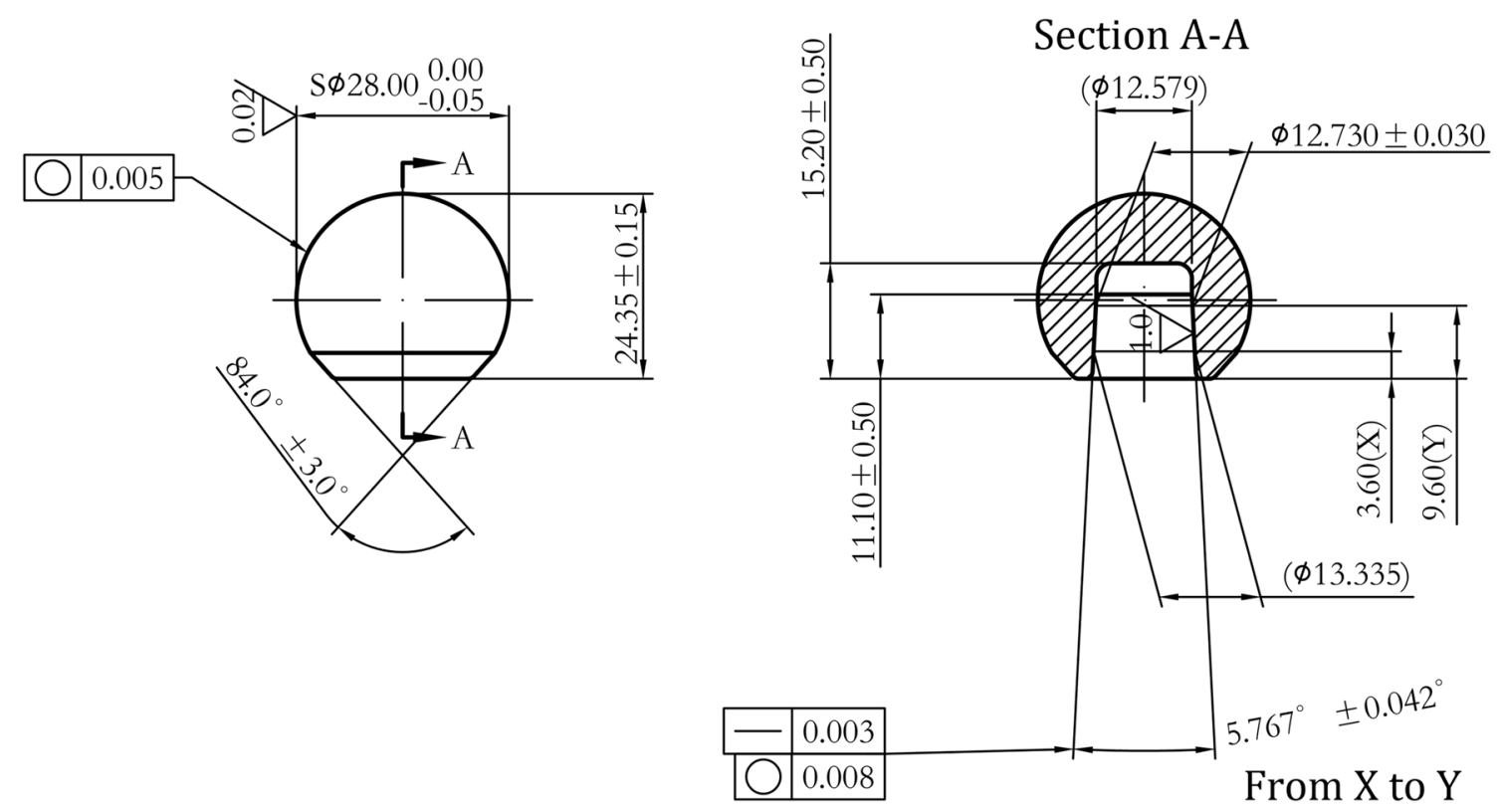

Fig. 3 Dimension and tolerance of femoral head ( $\$ 28$ mm—Type L)

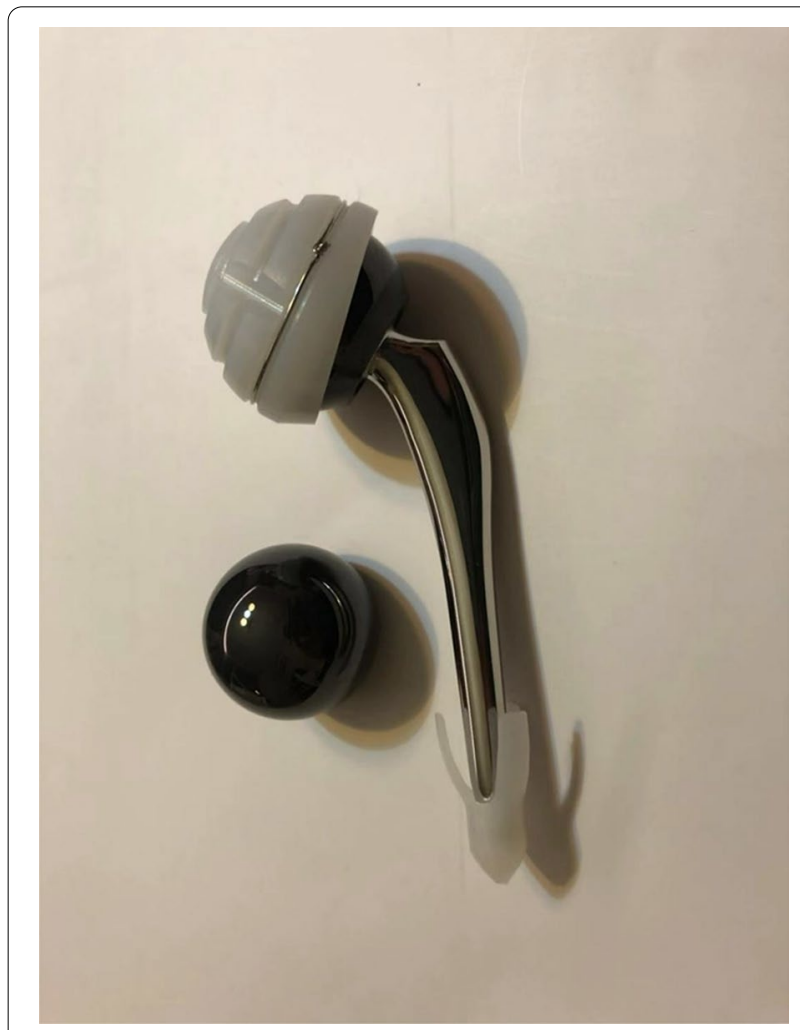

Fig. 4 Customized total hip system for pig

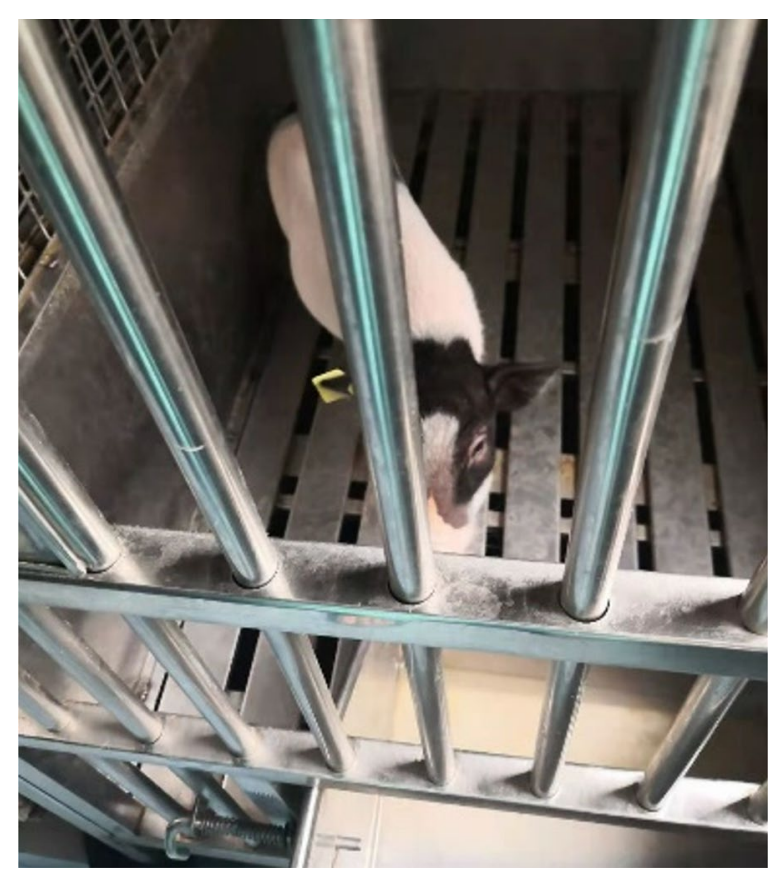

Fig. 5 Bama minipig involved in the experiment

unfortunately for ageing or accelerated ageing, there is no any standard or reference on which could be relied.

Several authors reported that the ageing zirconia could be achieved by means of autoclave treatment at $134{ }^{\circ} \mathrm{C}$ and 2 bars of pressure. Under this configuration, 
it was calculated that $1 \mathrm{~h}$ exposure had theoretically the similar effect as roughly 3-4 years in the human body.

In this study, the silicon nitride sample bars (30 pieces) and eight femoral heads had been aged via $10 \mathrm{~h}$ autoclave treatment $\left(134{ }^{\circ} \mathrm{C}, 2\right.$ bars $)$ for the following tests.

\section{Elastic moduli test}

According to (ISO 17561), ten specimens unaged had been tested at room temperature for determining the Young's modulus by sonic resonance.

\section{Flexural strength test}

As per (ISO 14704), ten specimens unaged with another ten specimens aged by using process defined in Sect. 2.2.1 had been investigated correspondingly for obtaining the average flexural strength in four-point- $1 / 4$ point flexure with $40 \mathrm{~mm}$ span semi-articulating fixtures and the bearings were free to roll.

\section{Fracture toughness test}

Considering the material proprieties, the notch feasibility and accuracy, the indentation fracture method had been adopted for evaluating the fracture resistance of silicon nitride. In accordance with (ISO 21618), ten specimens unaged and aged had been examined, respectively, with an indentation load of $98.07 \mathrm{~N}$.

\section{Fatigue strength test}

As a pass/fail test for determination of the overall fatigue behavior, consistent with (ISO 22214), five specimens unaged and aged had been carried out separately at room temperature in air $\left(25 \pm 5{ }^{\circ} \mathrm{C}, \mathrm{RH} 40 \pm 10 \%\right)$. The fourpoint- $1 / 4$ point flexure with $40 \mathrm{~mm}$ span semi-articulating fixtures had been used. The waveform of loading stress is a sinusoidal wave of $10 \mathrm{~Hz}$ frequency and a stress ration $\mathrm{R}=0.1$. The minimum and maximum force applied were $50 \mathrm{~N}$ and $500 \mathrm{~N}$, respectively. The number of cycles for suspension was set at 10 million.

\section{Vickers hardness test}

Refer to (ISO 14705), five specimens unaged and aged had been inspected respectively for determining the Vickers hardness at room temperature in air. The test force used was $98.07 \mathrm{~N}$.

\section{Cytotoxicity test}

According to (ISO 10993-5), in this study, the end-point measured in cytotoxicity determination was the measurement of cell growth via the extract test. The related materials and methods were detailed in the "Appendix" due to the constraint on length of body text.
Salmonella typhimurium reverse mutation assay/Ames test (OECD_Organization for Economic Co-operation and Development 471)

As per (ISO 10993-3) and OECD 471 guideline, the specimen's genotoxicity, gene mutations in bacteria, its mutagenicity had been investigated via the number of colonies observed. The related materials and methods were detailed in the "Appendix" due to the constraint on length of body text.

\section{TK gene mutation test using mouse lymphoma cells (OECD} 476)

Refer to (ISO 10993-3) and OECD 476 guideline, the specimen's genotoxicity, gene mutations in mammalian cells, had been evaluated via the observation of mouse lymphoma cells (L5178Y TK ${ }^{ \pm}-3.7 .2 \mathrm{C}$ )' forward mutation. The related materials and methods were detailed in the Appendix due to the constraint on length of body text.

\section{Mammalian chromosome aberration test (OECD 473)}

In accordance with (ISO 10993-3) and OECD 473 guideline, the specimen's genotoxicity, clastogenicity in mammalian cells, had been examined via the inducement of chromosome aberration in the Chinese Hamster Lung (CHL) cells. The related materials and methods were detailed in the Appendix due to the constraint on length of body text.

\section{In vivo experiments \\ Acute and sub chronic systemic toxicity test}

According to (ISO 10993-11), for the acute systemic toxicity test, 20 mice randomized in 4 groups had been involved, the related materials and methods were detailed in the "Appendix" due to the constraint on length of body text.

\section{Irritation test}

As per (ISO 10993-10), with regard to the test material, its potential to produce irritation had been assessed via the animal skin irritation test. The related materials and methods were detailed in the "Appendix" due to the constraint on length of body text.

\section{Delayed-type hypersensitivity test}

Refer to (ISO 10993-10), for the silicon nitride in this study, its delayed-type hypersensitivity had been evaluated via the guinea pig maximization test. The related materials and methods were detailed in the Appendix due to the constraint on length of body text.

\section{Test for local effects after implantation in bone}

In accordance with (ISO 10993-6), the local effects after implantation had been investigated via the implantation 
test in rabbit femur with cylindrical implants $2 \mathrm{~mm}$ in diameter and $6 \mathrm{~mm}$ in length. The related materials and methods were detailed in the "Appendix" due to the constraint on length of body text.

\section{Total hip implantation test in pigs}

Three mini pigs were randomly chosen and involved in preliminary experiment for being proficient in approach and surgical technique. And then, ten Bama minipigs were randomly divided into the test group (with total hip arthroplasty) and control group (with arthrotomy) regardless the gender.

Before surgery, a CT scan was accomplished for ensuring its anatomical status. The general anesthesia and trachea cannula were employed. The operating side was randomized on hind legs, a lateral approach was adopted. After disinfection and draping, lateral muscles were cut to expose the hip joint. Then, a swing saw was used to cut off femoral neck, then the femoral head was removed and the acetabulum was exposed. The appropriate acetabular reamer was used to clear acetabulum. And a dislocation hook was used to lift femur and medullary space was prepared. After these preparation, acetabular cup impacted and femoral stem inserted, they were fixed by bone cement. The silicon nitride ceramic femoral head was implanted subsequently. After testing the stability of the joint, the incision was closed layer by layer. The blank control group finished arthrotomy with the same approach (Fig. 6).

After surgery, in order to reduce the risk of blood loss, infection, and pain release, tranexamic acid $\left(1 \mathrm{~g} 100 \mathrm{ml}^{-1}\right.$ per os), ceftriaxone sodium (0.25 g intramuscular injection), and pethidine ( $2 \mathrm{mg} \mathrm{kg}^{-1}$ intramuscular injection) were applied. The clinical observations had been made weekly, the hematology and clinical biochemical determination on blood had been analyzed and recorded monthly. The second CT scan had been accomplished just before the animal euthanasia which was carried out at the end of three months after the implantation, whereafter, the pathology examination had been conducted.

\section{Results}

According to the related ISO standards, by using the materials and methods detailed in Sect. 2, the results obtained are summarized in the relevant tables and paragraphs hereunder.

\section{In vitro experiments \\ Elastic moduli test}

\begin{tabular}{ll}
\hline Specimen no & $\begin{array}{l}\text { Young's } \\
\text { modulus } \\
\text { (GPa) }\end{array}$ \\
\hline 01 & 296 \\
02 & 301 \\
03 & 300 \\
04 & 302 \\
05 & 296 \\
06 & 294 \\
07 & 302 \\
08 & 302 \\
09 & 301 \\
10 & 297 \\
Mean & 299 \\
SD & 3 \\
\hline
\end{tabular}

As a comparable reference, the current mainstream product, BIOLOX ${ }^{\circledR}$ delta, from CeramTec, its Young's modulus should be $\geq 320 \mathrm{GPa}$ according to ISO 6474-2 for Type X: extra-high strength. And, the lower elastic modulus is usually considered as a favorable factor for stress shielding reduction.

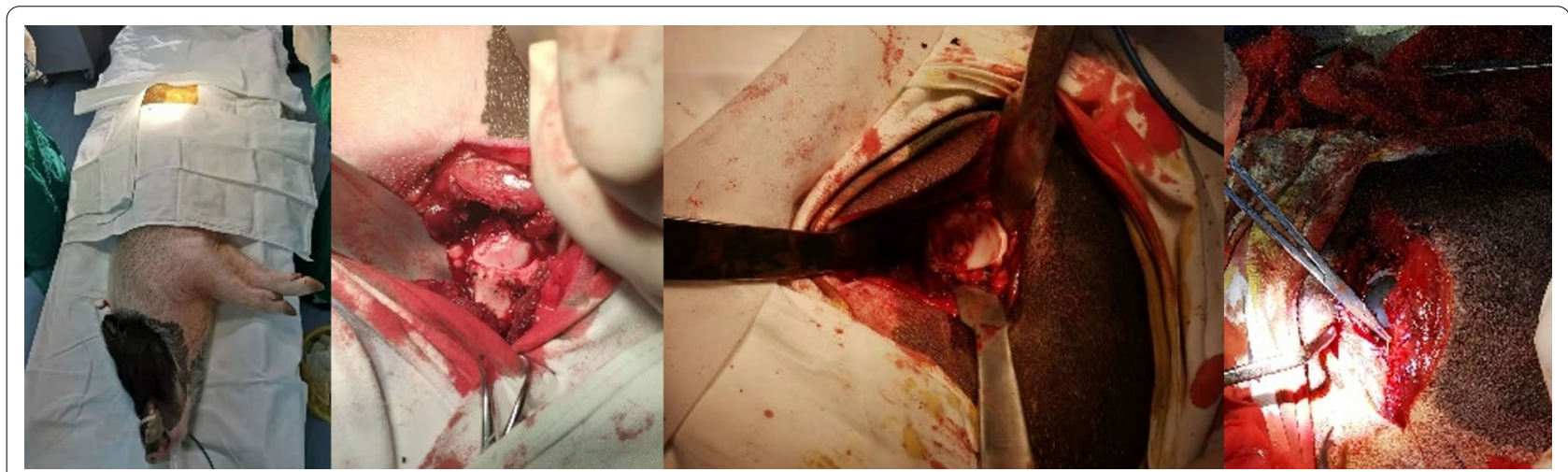

Fig. 6 Total hip implantation in pig. From left to right: lateral position, disinfection \& draping—hip joint exposure—acetabular preparation— prosthesis implantation 
Flexural strength test

\begin{tabular}{llll}
\hline $\begin{array}{l}\text { Specimen no. } \\
\text { (unaged) }\end{array}$ & $\begin{array}{l}\text { Flexural } \\
\text { strength } \\
\text { (MPa) }\end{array}$ & $\begin{array}{l}\text { Specimen no. } \\
\text { (aged) }\end{array}$ & $\begin{array}{l}\text { Flexural } \\
\text { strength } \\
\text { (MPa) }\end{array}$ \\
\hline 11 & 711 & 21 & 862 \\
12 & 790 & 22 & 782 \\
13 & 853 & 23 & 741 \\
14 & 815 & 24 & 733 \\
15 & 813 & 25 & 825 \\
16 & 725 & 26 & 808 \\
17 & 854 & 27 & 724 \\
18 & 756 & 28 & 790 \\
19 & 875 & 29 & 776 \\
20 & 770 & 30 & 840 \\
Mean & 796 & Mean & 788 \\
SD & 53 & SD & 44 \\
\hline
\end{tabular}

As per ISO $6474-2$, for BIOLOX $^{\circledR}$ delta, the mean 4-point flexural strength should be $\geq 750 \mathrm{MPa}$ and $1000 \mathrm{MPa}$ for Type S: standard high strength and Type $\mathrm{X}$ : extra-high strength respectively. It is worthwhile to mention that the sample bar's geometry, tolerance and surface condition including the chamfer and rounding are all crucial to the testing results. Around $1000 \mathrm{MPa}$ had been registered in another research project.

\section{Fracture toughness test}

\begin{tabular}{llll}
\hline $\begin{array}{l}\text { Specimen no. } \\
\text { (unaged) }\end{array}$ & $\begin{array}{l}\text { Fracture } \\
\text { toughness } \\
\text { (MPa m }^{\text {1/2) }}\end{array}$ & $\begin{array}{l}\text { Specimen no. } \\
\text { (aged) }\end{array}$ & $\begin{array}{l}\text { Fracture } \\
\text { toughness } \\
\left.\text { (MPa m }^{1 / 2}\right)\end{array}$ \\
\hline 31 & 6.05 & 41 & 6.35 \\
32 & 6.40 & 42 & 6.21 \\
33 & 6.33 & 43 & 6.29 \\
34 & 6.16 & 44 & 6.27 \\
35 & 6.19 & 45 & 6.36 \\
36 & 6.29 & 46 & 6.12 \\
37 & 6.25 & 47 & 6.16 \\
38 & 6.18 & 48 & 6.24 \\
39 & 6.36 & 49 & 6.26 \\
40 & 6.26 & 50 & 6.30 \\
Mean & 6.25 & Mean & 6.26 \\
SD & 0.10 & SD & 0.07 \\
\hline
\end{tabular}

According to ISO 6474-2, for BIOLOX ${ }^{\circledR}$ delta, the fracture toughness should be $\geq 3.5$ and $4.0 \mathrm{MPa} \mathrm{m}{ }^{1 / 2}$ for Type $\mathrm{S}$ and Type $\mathrm{X}$ respectively.

\section{Fatigue strength test}

On conditions detailed in Sect. 2.2.5, both unaged and aged specimens passed the 10 million loading cycles without any failure or intergroup difference either.

\section{Vickers hardness test}

\begin{tabular}{llll}
\hline $\begin{array}{l}\text { Specimen no. } \\
\text { (unaged) }\end{array}$ & Hardness (HV10) & $\begin{array}{l}\text { Specimen } \\
\text { no. (aged) }\end{array}$ & Hardness (HV10) \\
\hline 61 & 1426 & 66 & 1426 \\
62 & 1460 & 67 & 1436 \\
63 & 1460 & 68 & 1460 \\
64 & 1449 & 69 & 1426 \\
65 & 1460 & 70 & 1467 \\
Mean & 1451 & Mean & 1443 \\
SD & 13 & SD & 17 \\
\hline
\end{tabular}

In the light of ISO $6474-2$, for BIOLOX $^{\circledR}$ delta, the hardness, Vickers HV1, should be $\geq 15.5$ and $16.0 \mathrm{GPa}$ for Type $S$ and Type $X$, respectively.

\section{Cytotoxicity test}

On conditions detailed in Sect. 2.2.7, based upon the comparison with the positive, negative and reagent controls, the relative cell proliferation had been investigated and the qualitative evaluation had been carried out as well. Microscopically, in the majority of cells, there was no changes in general morphology, vacuolization, detachment and membrane integrity. Only few cell lysis had been observed. The cytotoxicity scale was consequently scored as 1 , mildly cytotoxic, which is totally acceptable for the intended use.

\section{Salmonella typhimurium reverse mutation assay/Ames test (OECD 471)}

On conditions detailed in Sect. 2.2.8, comparing with the reagent controls, the inhibition of 5 types of salmonella typhimurium had not been observed in the test groups with polar or non-polar solvents. And therefore the specimen's genotoxicity, gene mutations in bacteria, was negative.

\section{TK gene mutation test using mouse lymphoma cells (OECD 476)}

On conditions detailed in Sect. 2.2.9, the reagent controls was used as the baseline, with regard to the mouse lymphoma cells forward mutation, the mutant frequency ratio of the test groups with polar or nonpolar solvents and the reagent controls was inferior to 
2. And, the specimen's genotoxicity, gene mutations in mammalian cells, was consequently negative.

\section{Mammalian chromosome aberration test (OECD 473)}

On conditions detailed in Sect. 2.2.10, according to the response of reagent controls, regarding the chromosome aberration in CHL cells, there was no statistical difference $(p>0.05)$ between the test groups and the reagent controls. And accordingly, the specimen's genotoxicity, clastogenicity in mammalian cells, was negative.

\section{In vivo experiments}

\section{Acute \& sub chronic systemic toxicity test}

Under the circumstances detailed in Sect. 2.3.1, regardless of the test groups with polar/non-polar solvents or reagent controls, the clinical observation was all normal for each mouse at the different inspection points. And during the observation period, all the body weights were increased and the mortality was zero. In one word, the specimen's acute systemic toxicity was negative.

For the subchronic systemic toxicity, in accordance with the reagent controls, in terms of the clinical observation, bodyweight change, hematology, clinical biochemical determination on blood, gross pathology and histopathology, there was no statistical difference $(p>0.05)$ between the test and control groups. As a result of which, the specimen's subchronic systemic toxicity was negative.

\section{Irritation test}

Under the circumstances detailed in Sect. 2.3.2, irrespective as to whether test groups with polar/non-polar solvents or reagent controls, no any erythema or eschar or edema occurred, upon which, the specimen's response to irritation test was negative.

\section{Delayed-type hypersensitivity test}

Under the circumstances detailed in Sect. 2.3.3, regardless of the test groups with polar/non-polar solvents or reagent controls, the clinical observation was all normal for each Hartley albino guinea pig during the observation period. There was no any erythema or edema observed. Based upon the patch test reactions, according to the Magnusson \& Kligman scale, the grading score obtained was less than 1 . Consequently, the specimen's response to delayed-type hypersensitivity test was negative.

\section{Test for local effects after implantation in bone}

Under the circumstances detailed in Sect. 2.3.4, for both the test and control group, there was no abnormal local effects observed through the 26 weeks after the implantation. 1 week after implantation, a few fibrous osteoid callus were similarly observed around the test and control

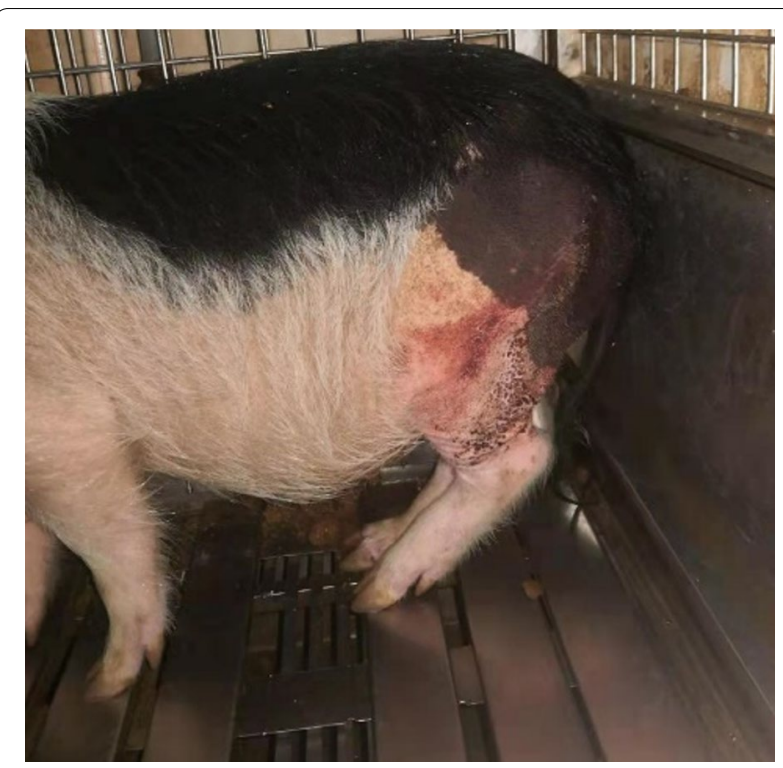

Fig. 7 Postoperative posture

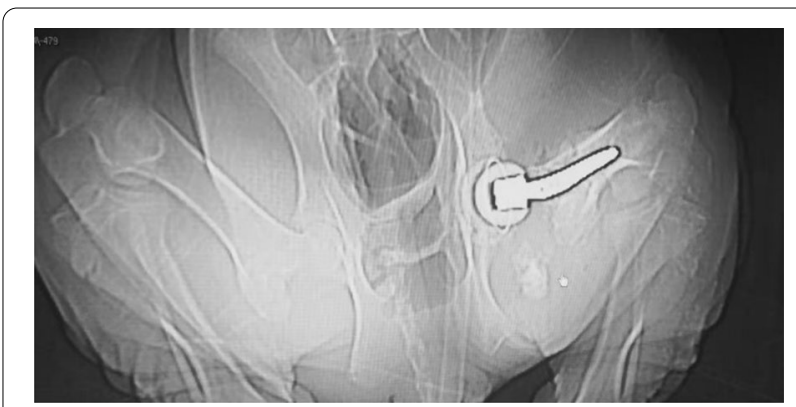

Fig. 8 CT scan result (test group, No. 4 pig), prone position

sample. 4 weeks after the implantation, the test and control specimen engaged closely with bone tissue, cell proliferation and trabeculae were observed equally. 26 weeks after the implantation, the new bone formed healthily around the test and control sample, the densified cortical bone were observed, there was no any foreign body reaction. In conclusion, the specimen's response to local effects after implantation in bone was negative.

\section{Total hip implantation test in pigs}

Under the circumstances detailed in Sect. 2.3.5, comparing with the control group, all the samples in test group were functioning well through the observation period (Fig. 7). The blood test results were normal, in terms of the $\mathrm{C}$-reactive protein and erythrocyte sedimentation rate, there was no difference between the test and control group. The CT scan results reveal the prostheses were on good position (Fig. 8). And the pathology examination 
results were negative. More detailed information could be found in another clinical report.

\section{Discussion}

This study presents a thorough scenograph, from material level to product level, of the specific silicon nitride used as the femoral head in total hip implant system.

Comparing with the current monopolized product, BIOLOX $^{\circledR}$ delta femoral head from CeramTec (Plochingen, Germany), this ZTA product's performance is regularized by (ISO 6474-2), Black Ceramic ${ }^{\circledR}$ silicon nitride femoral head has the comparable mechanical performance.

Meanwhile, the latter's fracture toughness is even better, the density is lower, the related implant will be lighter consequently, and exclusively, without the concern about the zirconia's aging and instability, which are caused by the inherent and inevitable phase transformation.

Based upon the current test results obtained, the silicon nitride in this study has a superior oxidation resistance since its mechanical performances are almost indistinguishable between the unaged and aged group.

In terms of the limitation of this study, although the accelerated ageing method adopted will permit an investigator to compare the oxidative stability of silicon nitride, it is recognized that it may not precisely simulate the degradative mechanisms for an implant during real time shelf ageing and implantation. And, concerning shelf ageing component, the package environment other than air is not taken into account, the related effect could be possibly not negligible.

In addition, for the ZTA or BIOLOX ${ }^{\circledR}$ delta, according to the ISO 6474-2, after autoclaving $(10 \mathrm{~h}, 0.2 \mathrm{MPa}$, $134{ }^{\circ} \mathrm{C}$ ), the $20 \%$ strength degradation is allowable. For the future studies, comparing with this silicon nitride, it will be interesting to know their performances evolution with higher pressure, temperature and longer ageing process. It is important to emphasize that, comparing with the metastable oxide ceramics, the silicon nitride could withstand more severe ageing method without performance degradation due to its unique, innate and outstanding integrated characteristics.

Another gap needs to be filled, in the orthopedic domain, the implant's wear performance is crucial as well. Although the silicon nitride is well known as a selflubricated material, its wear resistance as the hip joint prosthesis still needs to be demonstrated in vitro according to ISO 14242 which is also the regulatory prerequisite. This will be the next step in the near future.

There are several simulator studies have been accomplished [13-15]. Recently, Affatato et al. accomplished the first in vitro wear behavior comparison between silicon nitride and ZTA head, the results are very promising [16].

With regard to the first ceramic total hip replacement in big animal, this test is a complementary validation of the safety and effectiveness of this silicon nitride ceramic, three months observation, it is definitely not long enough for investigating its wear performance in vivo, which is the reason for why to continue the animal study and to deploy the appropriate human clinical trial as well for the future researches.

However, in the context of (ISO 14242-1), among the current simulated conditions, it is worthwhile to investigate the more realistic wear performance under the slow-speed, high-loads with shock and interrupted (stopstart) conditions, in addition, more important, the edge loading, ageing and wearing are occurred simultaneously [17]. In this respect, beyond the metastable oxide ceramics, along the echelon of advanced technical ceramics, looking at, the non-oxide, real stable ceramic, the silicon nitride, its potential and advantages will be highlighted contrastively in the future investigations.

Furthermore, G. Pezzotti et al. revealed that the oxidation rate of polyethylene liners was greater when coupled with oxide as opposed to non-oxide ceramic heads [18-20]. And, in addition, J. Webster et al. discovered for the first time that without the use of antibiotics, the bacteria colonization was reduced and bone formation was increased on the silicon nitride surface compared with titanium and PEEK [21-23]. These two lighthouses illumine also the meaningful directions for the future exploration.

\section{Conclusion}

Through all these in vitro \& in vivo experiments, this silicon nitride hip implant's safety and effectiveness have been investigated.

Comparing with the present metastable oxide ceramics, silicon nitride has the right combination of high strength, high hardness, excellent fracture toughness, lower density, better wear resistance, good biocompatibility, inherent stability, corrosion resistance and bioactivity, bone integration capability, all of which are desirable in medical implants.

Due to the excellent combination of properties, this silicon nitride will be an excellent material that can withstand the toughest conditions in the most demanding applications like in orthopedic and beyond.

\section{Appendix: Test materials and methods used Cytotoxicity test}

According to (ISO 10993-5), in this study, the endpoint measured in cytotoxicity determination was the 
measurement of cell growth via the extract test. For the extraction vehicle, the solvent used was the culture medium with $10 \%$ calf serum. The extraction condition was $24 \pm 2 \mathrm{~h}$ with $60 \mathrm{rpm}$ agitation at $37 \pm 1{ }^{\circ} \mathrm{C}$ with $2.3 \mathrm{~g}$ specimen in $11.5 \mathrm{ml}$ culture solution. About $10 \%$ dimethyl sulfoxide (DMSO) solution was selected as the positive control. The negative control material was polyethylene, its extraction condition was $24 \pm 2 \mathrm{~h}$ with $60 \mathrm{rpm}$ agitation at $37 \pm 1{ }^{\circ} \mathrm{C}$ by an interfacial area-volume ratio of $6 \mathrm{~cm}^{2} \mathrm{ml}^{-1}$ culture solution. The extraction vehicles without test material subjected to the same extraction conditions were used as the reagent control. The cell lines used were American Type Culture Collection CCL 1 (NCTC-National Collection of Type Cultures clone 929). Incubation time was $72 \mathrm{~h}\left(5 \% \mathrm{CO}_{2}, 37^{\circ} \mathrm{C}\right)$.

\section{Salmonella typhimurium reverse mutation assay/Ames test (OECD 471)}

As per (ISO 10993-3) and OECD 471 guideline, the specimen's genotoxicity, gene mutations in bacteria, its mutagenicity had been investigated via the number of colonies observed. The salmonella typhimurium TA97, TA98, TA100, TA102 and TA1535 had been used in this study. In terms of the extraction vehicle, the solvents used were $0.9 \%$ sodium chloride (SC) and DMSO. The extraction conditions were $1 \pm 0.1 \mathrm{~h}$ with $60 \mathrm{rpm}$ agitation at $121 \pm 2{ }^{\circ} \mathrm{C}$ with $6 \mathrm{~g}$ specimen in $30 \mathrm{ml} \mathrm{SC}$ injection and $72 \pm 2 \mathrm{~h}$ with $60 \mathrm{rpm}$ agitation at $37 \pm 1{ }^{\circ} \mathrm{C}$ with $2.8 \mathrm{~g}$ specimen in $14 \mathrm{ml}$ DMSO solution. The extraction vehicles without test material subjected to the same extraction conditions were used as the reagent control. Incubation time was $72 \mathrm{~h}\left(5 \% \mathrm{CO}_{2}, 37^{\circ} \mathrm{C}\right)$.

\section{TK gene mutation test using mouse lymphoma cells (OECD 476)}

Refer to (ISO 10993-3) and OECD 476 guideline, the specimen's genotoxicity, gene mutations in mammalian cells, had been evaluated via the observation of mouse lymphoma cells (L5178Y TK TK $^{ \pm}$3.7.2C)' forward mutation. With regard to the extraction vehicle, the solvents used were $0.9 \% \mathrm{SC}$ injection and DMSO solution. The extraction conditions were $1 \pm 0.1 \mathrm{~h}$ with $60 \mathrm{rpm}$ agitation at $121 \pm 2{ }^{\circ} \mathrm{C}$ with $6 \mathrm{~g}$ specimen in $30 \mathrm{ml} \mathrm{SC}$ injection and $72 \pm 2 \mathrm{~h}$ with $60 \mathrm{rpm}$ agitation at $37 \pm 1{ }^{\circ} \mathrm{C}$ with $2.8 \mathrm{~g}$ specimen in $14 \mathrm{ml}$ DMSO solution. The extraction vehicles without test material subjected to the same extraction conditions were used as the reagent control. The calf serum and RPMI (Roswell Park Memorial Institute)-1640 had been used as medium. Incubation time was 14 days $\left(5 \% \mathrm{CO}_{2}, 37^{\circ} \mathrm{C}\right)$.
Mammalian chromosome aberration test (OECD 473)

In accordance with (ISO 10993-3) and OECD 473 guideline, the specimen's genotoxicity, clastogenicity in mammalian cells, had been examined via the inducement of chromosome aberration in the Chinese hamster lung (CHL) cells. Regarding the extraction vehicle, the solvents used were $0.9 \% \mathrm{SC}$ injection and DMSO solution. The extraction conditions were $1 \pm 0.1 \mathrm{~h}$ with $60 \mathrm{rpm}$ agitation at $121 \pm 2{ }^{\circ} \mathrm{C}$ with $6 \mathrm{~g}$ specimen in $30 \mathrm{ml} \mathrm{SC}$ injection and $72 \pm 2 \mathrm{~h}$ with $60 \mathrm{rpm}$ agitation at $37 \pm 1{ }^{\circ} \mathrm{C}$ with $2.8 \mathrm{~g}$ specimen in $14 \mathrm{ml}$ DMSO solution. The extraction vehicles without test material subjected to the same extraction conditions were used as the reagent control. The calf serum and RPMI-1640 had been used as medium. Incubation time was $78 \mathrm{~h}\left(5 \% \mathrm{CO}_{2}, 37^{\circ} \mathrm{C}\right)$ totally.

\section{Acute and sub chronic systemic toxicity test}

According to (ISO 10993-11), for the acute systemic toxicity test, 20 mice randomized in 4 groups had been involved, initial weight between 17 and $23 \mathrm{~g}$, aged between 6 and 8 weeks. For the extraction vehicle, the solvents used were $0.9 \% \mathrm{SC}$ injection and cottonseed oil (CSO). The extraction conditions were $1 \pm 0.1 \mathrm{~h}$ with $60 \mathrm{rpm}$ agitation at $121 \pm 2{ }^{\circ} \mathrm{C}$ with $2.4 \mathrm{~g}$ specimen in $12 \mathrm{ml} \mathrm{SC}$ and $2.3 \mathrm{~g}$ specimen in $11.5 \mathrm{ml} \mathrm{CSO}$. The extraction vehicles without test material subjected to the same extraction conditions were used as the reagent control. The routes of administration were intravenous (IV) for SC groups and intraperitoneal (IP) for CSO groups, respectively. The dosage level was $50 \mathrm{ml} \mathrm{kg}^{-1}$. The clinical observations had been made immediately after dosing and $4 \mathrm{~h}, 24 \mathrm{~h}, 48 \mathrm{~h}$ and $72 \mathrm{~h}$. The body weight measurements had been accomplished at $24 \mathrm{~h}, 48 \mathrm{~h}$ and $72 \mathrm{~h}$ correspondingly.

With regard to the sub chronic systemic toxicity test, as per the sex, 40 rats randomized in 4 groups had been involved, initial weight range was 191-215 g for female and 211-239 g for male, age range 6-9 weeks. For the extraction vehicle, the solvent used was $0.9 \%$ SC injection. The extraction condition was $72 \pm 2 \mathrm{~h}$ with $60 \mathrm{rpm}$ agitation at $37 \pm 1{ }^{\circ} \mathrm{C}$ with a ratio of $0.2 \mathrm{~g} \mathrm{ml}^{-1}$. The extraction vehicles without test material subjected to the same extraction conditions were used as the reagent control. The route of administration was IV in a daily basis for consecutive 28 days. The dosage volume was 10 $\mathrm{ml} \mathrm{kg} \mathrm{kg}^{-1 *}$ BW-Body Weight. The clinical observations had been made every day, the body weight had been recorded weekly. The hematology and clinical biochemical determination on blood, gross pathology and histopathology had been carried out at the end of the test period. 


\section{Irritation test}

As per (ISO 10993-10), with regard to the test material, its potential to produce irritation had been assessed via the animal skin irritation test. Three healthy young adult albino rabbits had been randomly chosen and used. For the extraction vehicle, the solvents used were $0.9 \%$ $\mathrm{SC}$ injection and CSO. The extraction conditions were $1 \pm 0.1 \mathrm{~h}$ with $60 \mathrm{rpm}$ agitation at $121 \pm 2{ }^{\circ} \mathrm{C}$ with $2.8 \mathrm{~g}$ specimen in $14 \mathrm{ml} \mathrm{SC}$ and $3.2 \mathrm{~g}$ specimen in $16 \mathrm{ml} \mathrm{CSO}$. The extraction vehicles without test material subjected to the same extraction conditions were used as the reagent control. At the cranial end, five sites, on each side of the spine, had been selected for SC group and its control. And for the same animal, at the caudal end, 10 sites in total had been selected for CSO group and its control on both sides of the spine. The dosage volume was $0.2 \mathrm{ml}$ at each test and control site by intradermal injection. The clinical observations had been made immediately after dosing and $24 \pm 2 \mathrm{~h}, 48 \pm 2 \mathrm{~h}$ and $72 \pm 2 \mathrm{~h}$. The potential erythema or eschar or edema should be recorded correspondingly.

\section{Delayed-type hypersensitivity test}

Refer to (ISO 10993-10), for the silicon nitride in this study, its delayed-type hypersensitivity had been evaluated via the guinea pig maximization test. 30 Hartley albino guinea pigs, bodyweight range from 300 to $400 \mathrm{~g}$, had been randomly chosen and involved. For the extraction vehicle, the solvents used were $0.9 \%$ SC injection and CSO. The extraction conditions were $1 \pm 0.1 \mathrm{~h}$ with $60 \mathrm{rpm}$ agitation at $121 \pm 2{ }^{\circ} \mathrm{C}$ with $2 \mathrm{~g}$ specimen in $10 \mathrm{ml} \mathrm{SC}$ or CSO. The 1-Chloro-2, 4-dinitrobenzene was selected as the positive control, and the extraction vehicles without test material subjected to the same extraction conditions were used as the reagent control.

For the intradermal induction phase, a pair of $0.1 \mathrm{ml}$ of each of the following, into each animal, had been made at the injection sites (A, B and $\mathrm{C}$ ) as shown in Fig.

9 in the clipped intrascapular region.

Site A: a 50:50 (volume ration) stable emulsion of Freund's complete adjuvant (FCA) mixed with the chosen solvent.

Site B: the test sample (undiluted extract), the solvent alone for the control animals.

Site C: the test sample at the concentration used at site $\mathrm{B}$, emulsified in a 50:50 (volume ration) stable FCA emulsion and the chosen solvent (50\%), the emulsion of the blank liquid with FCA for the control animals.

Seven days after completion of the intradermal induction phase, the topical induction phase was started by using a patch of area approximately $8 \mathrm{~cm}^{2}$ filter paper (soaked in the concentration selected for site B) to cover the intradermal injection sites. These area had been

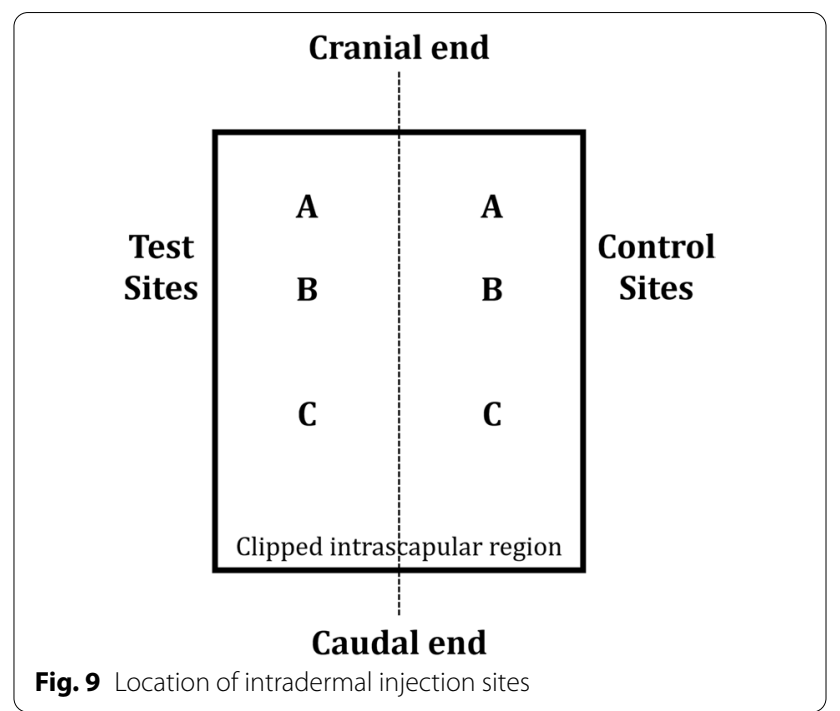

pretreated with $10 \%$ sodium dodecyl sulfate massaged into the skin $24 \pm 2 \mathrm{~h}$ before the patch was applied. The patches were secured with an occlusive dressing and these dressing and patches were removed after $48 \pm 2 \mathrm{~h}$. The control animals were treated similarly by using the reagent control alone.

At 14 days after completion of the topical induction phase, the challenge phase was kicked off by using the patches (soaked in the concentration selected for site $\mathrm{C}$ ) on the upper flank of each animal. After $24 \pm 2 \mathrm{~h}$, these patches and dressings were removed.

$24 \mathrm{~h}$ and $48 \mathrm{~h}$ after the removal of the dressings, the appearance of the challenged skin sites of the test and control animals were observed and the skin reactions for erythema and edema were recorded according to the Magnusson \& Kligman grading.

\section{Test for local effects after implantation in bone}

In accordance with (ISO 10993-6), the local effects after implantation had been investigated via the implantation test in rabbit femur with cylindrical implants $2 \mathrm{~mm}$ in diameter and $6 \mathrm{~mm}$ in length. The UHMWPE bars with same shape were used as the control implants. There were twenty rabbits randomly chosen and involved in this study. Two implantation sites were chosen on each rabbit femur and the control specimens were implanted contralaterally in the equivalent anatomical sites. The animal euthanasia was carried out respectively at 1 week, 4 weeks and 26 weeks after the implantation, and retrieval of the implant together with sufficient unaffected surrounding tissue. The relevant biological response was evaluated by documenting the macroscopic and histopathological responses as a function of time. 


\section{Abbreviations}

ISO: International Organization for Standardization; THA: Total Hip Arthroplasty; FDA: U. S. Food and Drug Administration; ZTA: Zirconia Toughened Alumina; 3Y-TZP: Yttria-stabilized Tetragonal Zirconia Polycrystal; CT: Computed Tomography; PLA: People's Liberation Army of China; UHMWPE: Ultra-High-Molecular-Weight-Polyethylene; OECD: Organization for Economic Co-operation and Development; $\mathrm{CHL}$ : Chinese Hamster Lung; DMSO: Dimethyl Sulfoxide; NCTC : National Collection of Type Cultures; SC: Sodium Chloride; RPMI: Roswell Park Memorial Institute; CSO: Cottonseed Oil; IV: Intravenous; IP: Intraperitoneal; BW: Body Weight; FCA: Freund's Complete Adjuvant.

\section{Acknowledgements}

All the silicon nitride components are offered by Black Ceramic Medical Technology Co., Ltd. (Suzhou, China), the metal femoral stems, acetabular cups are provided by LDK Technology Co., Ltd. (Beijing, China). Without the help from Shanghai Institute of Ceramics of the Chinese Academy of Sciences, Jinan Quality Supervision and Inspection Center for Medical Devices of National Medical Products Administration, Orthotek Laboratory (Shanghai, China) and Laboratory Animal Center of Chinese PLA General Hospital (Beijing, China), this work could not be accomplished, the authors would like to express the special thanks for their great contribution.

\section{Author contributions}

All authors have made substantial contributions to: (1) the conception and design of the study, (2) drafting the article or revising it critically for important intellectual content, (3) final approval of the version to be submitted. XK: Conceptualization, methodology, investigation, writing original draft, visualization, project administration. $\mathrm{XH}$ involved in methodology, investigation, writing original draft, visualization, animal handling. XK and XH contributed equally to this work. WC participated in conceptualization, methodology, investigation, resources, writing original draft, visualization, supervision, project administration, funding acquisition. All authors read and approved the final manuscript.

\section{Funding}

This study was funded by the National Natural Science Foundation of China (Grant-Number: 81772320)

\section{Availability of data and materials}

The datasets generated and/or analyzed during the current study are not publicly available due to the data also forms part of an ongoing study, but are available from the corresponding author on reasonable request.

\section{Declarations}

\section{Ethics approval and consent to participate}

All animal care and procedures were approved by the Institutional Review Board / Animal Care and Use Committee of Chinese PLA General Hospital and complied with the latest related guideline and the relevant ethics approval is obtained.

\section{Consent for publication}

Not applicable.

\section{Competing interests}

Co-first author, Xiaosu Hu is the CEO of Suzhou Black Ceramic Medical Technology Co., Ltd. The other authors declare that they have no known competing financial interests or personal relationships that could have appeared to influence the work reported in this paper.

\section{Author details}

${ }^{1}$ Senior Department of Orthopedics, The Fourth Medical Center of PLA general hospital, No. 51 Fucheng Road, Haidian, Beijing 100853, China. ${ }^{2}$ Black Ceramic Medical Technology Co., Ltd, No. 269, Dongjin Road, Wuzhong, Suzhou 215000, China.

Received: 5 November 2021 Accepted: 8 December 2021 Published online: 12 February 2022

\section{References}

1. Yang G, et al. Feasibility and safety of 2-day discharge after fast-track total hip arthroplasty: a Chinese experience. J Arthroplasty. 2016;31(8):16861692.e1. https://doi.org/10.1016/j.arth.2016.02.011.

2. Gaikwad S, et al. Evaluation of clinical outcome of cementless ceramic on ceramic primary total hip replacement in young patients of avascular necrosis of femoral head. J Arthrosc Joint Surg. 2020. https://doi.org/10. 1016/j.jajs.2020.11.004.

3. Charnley J. Arthroplasty of the hip. A new operation. Lancet. 1961. https://doi.org/10.1016/s0140-6736(61)92063-3.

4. Boutin P. Total arthroplasty of the hip by fritted alumina prosthesis. Experimental study and 1st clinical applications. Orthopaedics Traumatol Surg Res. 2014;100(1):15-21. https://doi.org/10.1016/j.rcot.2013.12.006.

5. https://www.ceramtec-medical.com/en/biolox

6. Sonny Bal B, et al. Ceramic materials in total joint arthroplasty. Sem Arthroplast. 2006;17(3-4):94-101. https://doi.org/10.1053/j.sart.2006.09. 002.

7. Christel $P$, et al. Biomechanical compatibility and design of ceramic implants for orthopedic surgery. Ann N Y Acad Sci. 1988;523:234-56. https://doi.org/10.1111/j.1749-6632.1988.tb38516.x.

8. Merola M, Affatato S. Materials for hip prostheses: a review of wear and loading considerations. Materials. 2019;12(3):495. https://doi.org/10.3390/ ma12030495.

9. https://www.fda.gov/inspections-compliance-enforcement-and-crimi nal-investigations/enforcement-story-archive/center-devices-and-radio logical-health-continued-2001

10. Ian CC. Metastable nature of zirconia femoral heads from a 20-year perspective of clinical and simulator wear studies. Sem Arthroplast. 2006;17(3-4):165-78. https://doi.org/10.1053/j.sart.2006.09.012.

11. Ian CC, et al. Current status of zirconia used in total hip implants. J Bone Joint Surg Am. 2003:85A(Suppl 4):73-84. https://doi.org/10.2106/00004 623-200300004-00009.

12. Chevalier J, Opinion L. What future for zirconia as a biomaterial? Biomaterials. 2006;27(4):535-43. https://doi.org/10.1016/j.biomaterials.2005.07. 034.

13. Sonny Bal B, et al. Testing of silicon nitride ceramic bearings for total hip arthroplasty. J Biomed Mater Res B Appl Biomater. 2008:87(2):447-54. https://doi.org/10.1002/jbm.b.31123.

14. Sonny Bal B, et al. Fabrication and testing of silicon nitride bearings in total hip arthroplasty winner of the 2007 "HAP" PAUL award. J Arthroplasty. 2009;24(1):110-6. https://doi.org/10.1016/j.arth.2008.01.300.

15. Clarke IC et al. Silicon nitride bearings: an alternative to oxide ceramics in total hip arthroplasty. Orthopaedic Proc. 2018;98-B(SUPP_1). https:// www.researchgate.net/publication/291189596_Silicon_nitride_beari ngs_an_alternative_to_oxide_ceramics_in_total_hip_arthroplasty

16. Yorifuji M, Affatato $S$, et al. Wear simulation of ceramic-on-crosslinked polyethylene hip prostheses: a new non-oxide silicon nitride versus the gold standard composite oxide ceramic femoral heads. Materials (Basel) 2020:13(13):2917. https://doi.org/10.3390/ma13132917.

17. Al-Hajjar M, et al. Combined wear and ageing of ceramic-on-ceramic bearings in total hip replacement under edge loading conditions. Materials. 2019;98:40-7. https://doi.org/10.1016/j.jmbbm.2019.05.011.

18. Pezzotti $G$, et al. On the molecular interaction between femoral heads and polyethylene liners in artificial hip joints: phenomenology and molecular scale phenomena. Biomed Mater. 2016;12(1):015005. https:// doi.org/10.1088/1748-605X/12/1/015005.

19. McEntire BJ, et al. Data-driven innovations in joint replacement: do ceramic femoral heads contribute to polyethylene oxidation? J Ceram Sci Tech. 2017;08(01):1-6. https://doi.org/10.4416/JCST2016-00100.

20. Pezzotti $G$, et al. Oxide ceramic femoral heads contribute to the oxidation of polyethylene liners in artificial hip joints. J Mech Behav Biomed Mater. 2018;82:168-82. https://doi.org/10.1016/j.jmbbm.2018.03.021.

21. Webster TJ, et al. Anti-infective and osteointegration properties of silicon nitride, poly(ether ether ketone), and titanium implants. Acta Biomater. 2012;8(12):4447-54. https://doi.org/10.1016/j.actbio.2012.07.038.

22. Gorth DJ, et al. Decreased bacteria activity on $\mathrm{Si}_{3} \mathrm{~N}_{4}$ surfaces compared with PEEK or titanium. Int J Nanomed. 2012;7:4829-40. https://doi.org/10. 2147/ijn.s35190. 
23. Akin SRK, et al. A comparative study of silicon nitride and SiAION ceramics against E. coli. Ceram Int. 2021;47(2):1837-43. https://doi.org/10. 1016/j.ceramint.2020.09.012

\section{Normative references}

24. "ISO 10993-10." Biological evaluation of medical devices—Part 10: Tests for irritation and skin sensitization. 2010. <https://www.iso.org/standard/ 40884.html>.

25. "ISO 10993-11." Biological evaluation of medical devices_Part 11: Tests for systemic toxicity. 2017. <https://www.iso.org/standard/68426.html>.

26. "ISO 10993-3." Biological evaluation of medical devices_-Part 3: Tests for genotoxicity, carcinogenicity and reproductive toxicity. 2014. <https://www. iso.org/standard/55614.html>.

27. "ISO 10993-5." Biological evaluation of medical devices_-Part 5: Tests for in vitro cytotoxicity. 2009. <https://www.iso.org/standard/36406.html>

28. "ISO 10993-6." Biological evaluation of medical devices_Part 6: Tests for local effects after implantation. 2016. <https://www.iso.org/standard/61089. html >.

29. "ISO 14242-1." Implants for surgery—Wear of total hip-joint prostheses-Part 1: Loading and displacement parameters for wear-testing machines and corresponding environmental conditions for test. 2014. <https://www.iso.org/ standard/63073.html>.

30. "ISO 14704." Fine ceramics (advanced ceramics, advanced technical ceramics) - Test method for flexural strength of monolithic ceramics at room temperature. 2016. <https://www.iso.org/standard/65411.html>.

31. "ISO 14705." Fine ceramics (advanced ceramics, advanced technical ceramics) - Test method for hardness of monolithic ceramics at room temperature. 2016. <https://www.iso.org/standard/65412.html>.

32. "ISO 17561." Fine ceramics (advanced ceramics, advanced technical ceramics) - Test method for elastic moduli of monolithic ceramics at room temperature by sonic resonance. 2012. <https://www.iso.org/standard/30748. html>.

33. "ISO 21618." Fine ceramics (advanced ceramics, advanced technical ceramics) - Test method for fracture resistance of monolithic ceramics at room temperature by indentation fracture (IF) method. 2019. <https://www.iso. org/standard/71248.html>.

34. "ISO 22214." Fine ceramics (advanced ceramics, advanced technical ceramics) - Test method for cyclic bending fatigue of monolithic ceramics at room temperature. 2006. <https://www.iso.org/standard/36167.html>.

35. "ISO 5834-3." Implants for surgery-UItra-high-molecular-weight polyethylene_Part 3: Accelerated ageing methods. 2019. <https://www.iso.org/ standard/73676.html>.

36. "ISO 6474-2." Implants for surgery—Ceramic materials—Part 2: Composite materials based on a high-purity alumina matrix with zirconia reinforcement. 2019. <https://www.iso.org/standard/72592.html>.

\section{Publisher's Note}

Springer Nature remains neutral with regard to jurisdictional claims in published maps and institutional affiliations.

Ready to submit your research? Choose BMC and benefit from:

- fast, convenient online submission

- thorough peer review by experienced researchers in your field

- rapid publication on acceptance

- support for research data, including large and complex data types

- gold Open Access which fosters wider collaboration and increased citations

- maximum visibility for your research: over $100 \mathrm{M}$ website views per year

At BMC, research is always in progress.

Learn more biomedcentral.com/submissions 